\title{
A 60GHz LTCC Transition between Microstrip Line and Substrate Integrated Waveguide
}

\author{
Tsung-Hsun Yang, Chi-Feng Chen, Ting-Yi Huang, Chun-Long Wang, and Ruey-Beei Wu \\ Department of Electrical Engineering and Graduate Institute of Communication Engineering, \\ National Taiwan University, Taipei, Taiwan 106, R.O.C \\ E-mail: rbwu@ew.ee.ntu.edu.tw
}

\begin{abstract}
The paper presents an open-circuited type transition between microstrip line and substrate integrated waveguide (SIW) fabricated on a low temperature co-fire ceramic (LTCC) substrate. The energy is coupled to the waveguide through a via probe from the feeding microstrip line. The stripline pad to the via probe is introduced to improve the transition performance. The structure height of $176 \mu \mathrm{m}$ which equals 0.073 guided wavelength is very short in microwave system. One single LTCC transition exhibits $10 \%$ bandwidth $(58-64 \mathrm{GHz}$ in the center frequency $60.5 \mathrm{GHz}$ ) for $-15 \mathrm{~dB}$ return loss and $2 \mathrm{~dB}$ insertion loss in the center frequency.
\end{abstract}

Index Terms- Microstrip line, LTCC, SIW, Waveguide transition.

\section{INTRODUCTION}

Planar transmission lines are prevailing in the microwave and millimeter-wave frequencies due to the advances of planar integrated technology. The low temperature co-fire ceramic (LTCC) technology can have the added feature of threedimensional interconnections. Combining the two structures can take great advantages of both structures, such as size down and mass production.

Among the planar transmission lines, microstrip line has easy integration with planar circuit devices. Nonetheless, the substrate integrated waveguide (SIW) attract increasing attention due to its multilayer and compact feature. Accordingly, many passive components are implemented, such as antenna feeds, high Q filters, diplexers, and so on. Thus, it is interesting to design transitions between microstrip line and SIW when integrating different circuit components.

Several transition designs have been reported, e.g., the transition is composed of a microstrip circuit, and in which a metal strip supported by the substrate serves as a probe to couple the energy from the waveguide [1]-[3]. An integrated LTCC laminated waveguide to microstrip line T-junction [4] and a tapered microstrip line to the top wall of the substrate integrated waveguide (SIW) [5]-[6]. In these designs, the microstrip line is shorted to either top or bottom walls of the waveguide, and meanwhile, some DC current which flows to metal walls of the waveguide would lead to power loss.

Hence, to solve this problem, it is helpful to have the feeding microstrip line open to the waveguide wall. This paper proposes a $60 \mathrm{GHz}$ LTCC transition between microstrip line and SIW. The planar transmission line is fed into the waveguide with via probe, but terminated with an open end. Adjusting the length of stripline pad could excite the maximal coupling. Numerical results are presented to verify design idea.

\section{DESCRIPTION OF TRANSITION}

Fig.1 shows the transition between the microstrip line and the SIW. It includes three parts: the microstrip line in the left hand side, the via probe with open stripline pad which is inserted on the $H$-plane, and the SIW which is formed by parallel vias and shorted on one side.

The via probe is roughly one quarter guided wavelength from the shorted wall so as to align vertically with the maximum $E$-field of the SIW. The stripline pad is a capacitance loading when its length is shorter than one quarter guided wavelength and an inductance loading when its length is from one quarter to half a guided wavelength. As a result, tuning the length of the stripline pad could improve the transition performance.

\section{THEORY}

The reciprocity theorem can be employed to calculate the modal amplitude of the $T E_{10}$ mode, given the total current $I_{v i a}$ along the via probe and the voltage $V_{v i a}$ on the via hole. Let the rectangular waveguide is of cross sectional size $a \times b$ and the height of the via probe is $h_{\text {via }}$ shown in Fig.1. After some algebraic elaboration, the power coupled to the $T E_{10}$ mode can be written by

$$
P_{10}=\frac{\omega \mu}{\beta_{10} a b} h_{v i a}^{2}\left|\left(I_{v i a}+j \omega C_{v i a} V_{v i a}\right)\right|^{2}
$$

where $C_{\text {via }} \approx \varepsilon A_{\text {hole }} / h_{\text {via }}$ in which $A_{\text {hole }}=\pi r_{\text {hole }}^{2}$ and $r_{\text {hole }}$ is the average radius of the via hole.

The contribution from $V_{v i a}$ is usually much smaller than that from $I_{\text {via }}$. Hence, more efficient coupling is possible if the via height is larger. However, the longer the via height is, the more manufacture space we need. Therefore, this paper proposes another way to increase the via current, for which tuning the length of the stripline pad is helpful. 

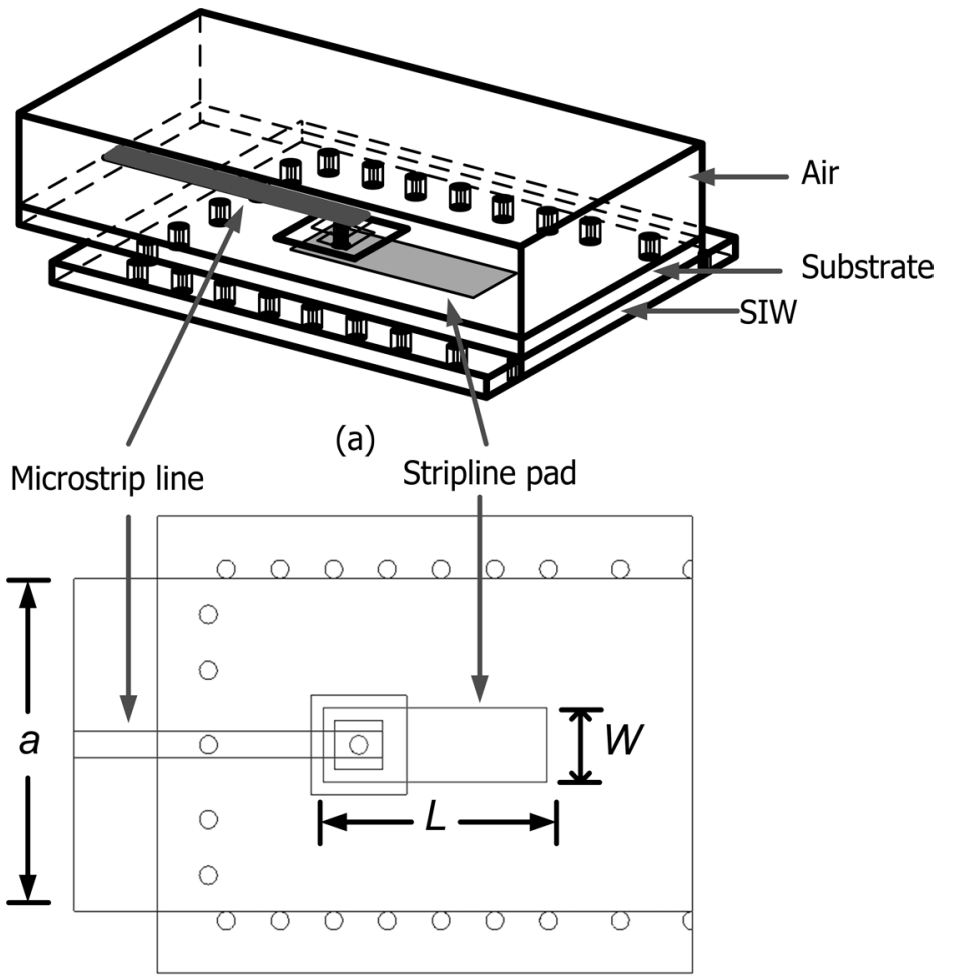

(b)

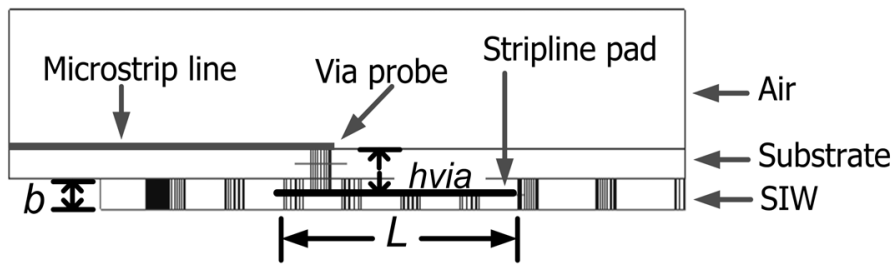

(c)

Fig.1 The structure of microstrip line to SIW transition with a via probe, (a) 3-D view (b) top view, and (c) side view.

\section{TRANSITION DESIGN}

To demonstrate the design procedure, consider a transition from a microstrip to SIW in $59-62 \mathrm{GHz}$. The relative dielectric constant of the SIW and substrate of microstrip line is $\varepsilon_{r}=7.8$, and loss tangent 0.02 . In order to satisfy the LTCC layer description and design rule, here, each LTCC layer has $44 \mu \mathrm{m}$ height. For the manufacturability, the via diameter is $75 \mu \mathrm{m}$, the conductor-to-conductor separation $100 \mu \mathrm{m}$, and the via-tovia separation $150 \mu \mathrm{m}$.

During the initial design, the waveguide side wall vias are replaced by perfect electric walls to speed up the simulation. They are added for more accurate simulation in the final design.
Consider first the transition between microstrip line to SIW. The design procedures are as follows.

1) To decide the width of the waveguide. For the center frequency of $60 \mathrm{GHz}$, the cutoff frequency of fundamental mode $T E_{10}$ is chosen to be $40 \mathrm{GHz}$. Since the cutoff frequency of second mode $T E_{20}$ will be $80 \mathrm{GHz}$, no higher order modes will be excited in $40-80 \mathrm{GHz}$. Given $\varepsilon_{r}$ and loss tangent at 60 $\mathrm{GHz}$, the waveguide width is chosen $a=1340 \mu \mathrm{m}$ and thus the guided wavelength $\lambda_{g}=1790 \mu \mathrm{m}$, propagation constant $\beta=2612 \mathrm{rad} / \mathrm{m}$, and attenuation constant $\alpha=47 \mathrm{~Np} / \mathrm{m}$.

2) To decide the width and length of the microstrip line. For input impedance match, a $50 \Omega$ feeding microstrip line is needed. Accordingly, tuning the width and length of the latter microstrip line could reduce the reflection, too.

3) To tune the width and length of the stripline pad. Fig.2 (a) shows the simulated return loss versus frequency with different lengths of the stripline pad. For $50 \Omega$ characteristic impedance, the width of the stripline pad equals $300 \mu \mathrm{m}$. The stripline pad resonates when the length of the stripline pad equals $936 \mu \mathrm{m}$, one half guided wavelength approximately. Meanwhile, the stripline pad excites the maximal coupling.

4) To obtain a final structure. The transition between microstrip line and SIW is shown in Fig. 1(c). The height of SIW is $88 \mu \mathrm{m}$ (two layers height), and the height of microstrip line substrate is $88 \mu \mathrm{m}$ (also two layers height). The structure of total height $176 \mu \mathrm{m}$ equals 0.073 guided wavelength is very short in much microwave system.

Four parameters, including length of the stripline pad, shape, width, and length of the feeding microstrip line, are adjusted one by one for better performance.

The thus obtained structure is analyzed by a full-wave field solver Ansoft HFSS version 8.5 and the simulated results are shown in Fig.2 (b). It is found that the transition has a good performance, with insertion loss $2 \mathrm{~dB}$ in $57-64 \mathrm{GHz}$ and return loss better than $-15 \mathrm{~dB}$ in $58-64 \mathrm{GHz}$. If dielectric loss is neglected, the simulation results show that the insertion loss is approximately zero in $59-62 \mathrm{GHz}$. Hence, the insertion loss can be improved significantly by adopting dielectric material of low dielectric loss tangent.

\section{CONCLUSION}

A transition between microstrip line and substrate integrated waveguide (SIW) is presented in this paper. This substrate integrated waveguide is located on a low temperature co-fire ceramic (LTCC) substrate. The transition structures from microstrip line to SIW are discussed.

One single LTCC designed transition exhibits $10 \%$ bandwidth for $-15 \mathrm{~dB}$ return loss and $2 \mathrm{~dB}$ insertion loss. Most of the insertion loss can be attributed to the dielectric loss of the substrate. Measurement for the back-to-back transition is in progress and will be presented in the conference. 


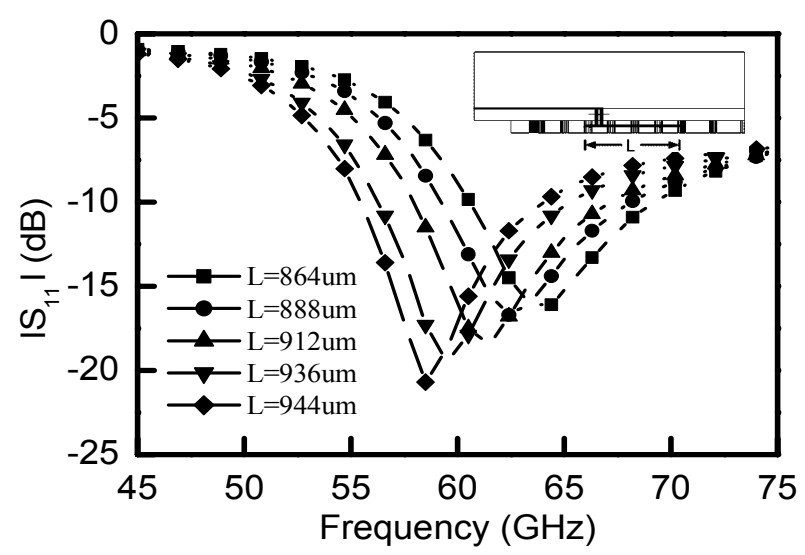

(a)

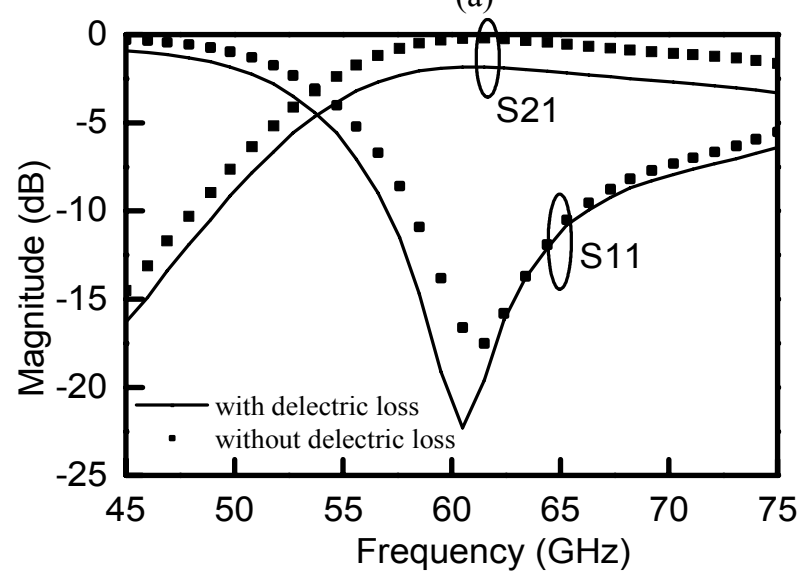

(b)

Fig.2 Simulated results of the transition structure. (a) Simulated return loss versus frequency with lengths of the stripline pad $\mathrm{L}$ as a parameter. (b) Simulated insertion and return loss versus frequency for the transition structure, with $a=1340 \mu \mathrm{m}, b=88 \mu \mathrm{m}, W=300 \mu \mathrm{m}, h_{v i a}=132 \mu \mathrm{m}$, and $L=936 \mu \mathrm{m}$. The solid and dashed curves denote the results with and without dielectric loss, respectively.

\section{ACKNOWLEDGMENT}

This work was supported in part by National Science Council, Taiwan, R.O.C. under Grant NSC 93-2752-E-002003-PAE.

\section{REFERENCES}

[1] Y. C. Shih, T. N. Ton, and L.Q. Bui, "Waveguide-to-microstrip transitions for millimeter-wave applications," IEEE MTT-S, Int. Microwave Symp. Dig., vol.1, pp. 473-475, May 1998.

[2] K. W. Kim, C.-H. Na, and D.-S. Woo, "New dielectric-cover waveguide-to-microstrip transitions for Ka-band transceivers," IEEE MTT-S, Int. Microwave Symp. Dig., vol.2, pp.1115-1118, June 2003.

[3] S. L.-Romano, B. P. Dorta-Naranjo, F. P.-Martinez, and M.S.Palma, "Ka-band waveguide-to-microstrip transitions design and implementation," IEEE Antennas and Propagation Society Symp., vol. 3, pp. 404-407, June 2002.

[4] Y. Huang, K.-L. Wu, and M. Ehlert, "An integrated LTCC laminated waveguide-to-microstrip line T-junction," IEEE Microwave Wireless Comp. Lett., pp.38-39, vol.13, Aug. 2003.

[5] D. Deslandes and K. Wu, "Integrated microstrip and rectangular waveguide in planar form," IEEE Microwave Wireless Comp. Lett., vol. 11, Feb. 2001.

[6] D. Deslandes and K. Wu, "Single-substrate integration technique of planar circuits and waveguide filters integrated microstrip and rectangular waveguide in planar form," IEEE Trans. Microwave Theory Tech., vol. 51, pp.338-339, Feb. 2003. 